\title{
On the Stability of Quadratic Functional Equation
}

\author{
Sushma \\ Department of Mathematics, \\ Kanya Mahavidyalaya Kharkhoda (India)
}

\begin{abstract}
In this paper the stability of quadratic functional equation, $f(x y)+f\left(x y^{-1}\right)=2 f(x)+2 f(y)$ on class of groups is obtained and also prove that quadratic functional equation may not be stable in any abelian group.
\end{abstract}

\section{Keywords}

Quadratic functional equation, pseudo-quadratic mapping, Banach space, quasi-quadratic mapping

\section{Mathematics Subject Classification Primary 54E40; Secondary 39B82, 46S50, 46S40}

\section{INTRODUCTION}

In 1940, Ulam[16] raised the following question concerning the stability of group homomorphism "Under what conditions does there is an additive mapping near an approximately additive mapping between a group and a metric group ?". In 1941, D.H.Hyers[10] answered the stability problem of Ulam under the assumption that the groups are Banach spaces. In 1950 Aoki[1] generalized the Hyers theorem for additive mappings. In 1978 Th.M.Rassias[14] provided a generalized version of the theorem of Hyers which permitted the Cauchy difference to become bounded. Since then, the stability problems of various functional equations have been extensively investigated by a number of authors [1-3,6,911].The functional equation $f(x+y)+f(x-y)=2 f(x)+2 f(y)$ is called a quadratic functional equation. In particular, every solution of quadratic functional equation is said to be a quadratic mapping. A generalized Hyers-Ulam stability problem for the quadratic functional equation was proved by Skof[15] for mappings $\mathrm{f}: \mathrm{X} \rightarrow \mathrm{Y}$ where $\mathrm{X}$ is a real normed space and $\mathrm{Y}$ is Banach space. Cholewa [4] noticed that the theorem of Skof[15] is still true if the relevant domain $\mathrm{X}$ is replaced by an Abelian group. Czerwik[5] and S.Y.Jang, J.R.Lee, C.Park and D.Y.Shin[12] proved the generalized Hyers-Ulam stability of the quadratic functional equation in Normed and Fuzzy Banach spaces respectively.

In this paper we study the stability of quadratic functional equation

$$
f(x y)+f\left(x y^{-1}\right)=2 f(x)+2 f(y) \text { on groups. }
$$

Suppose that $\mathrm{G}$ is an arbitrary group and $\mathrm{E}$ is an arbitrary real Banach space. We denote 1 the identity element of G.

Definition 2.1: We will say that a function $f: G \rightarrow E$ is a $(\mathrm{G} ; \mathrm{E})$ - Quadratic function if for any $x, y \in G$ we have

$$
f(x y)+f\left(x y^{-1}\right)-2 f(x)-2 f(y)=0(2.1)
$$

We denote the set of all (G; E) - Quadratic function by $Q(G ; E)$
Denote by $Q_{0}(G ; E)$ the subset of $Q(G ; E)$ consisting of functions $f$ such that $\mathrm{f}(1)=0$. Obviously $Q_{0}(G ; E)$ is a subspace of $Q(G ; E)$ and $Q(G ; E)=Q_{0}(G ; E) \oplus E$.

Definition 2.2: A function $f: G \rightarrow E$ is a $(G ; E)$ quasi - Quadratic function if there is $C>0$ such that for any $x, y \in G$ we have

$$
\left\|f(x y)+f\left(x y^{-1}\right)-2 f(x)-2 f(y)\right\| \leq c
$$

It is clear that the set of $(G ; E)$ - quasi - Quadratic functions is a linear space.

Denote it by $K Q(G ; E)$. In (2.2), we put $\mathrm{x}=1$,

$$
\begin{aligned}
& \left\|f(y)+f\left(y^{-1}\right)-2 f(y)-2 f(1)\right\|<c \\
& \left\|f\left(y^{-1}\right)-f(y)\right\|<c+2\|f(1)\|=c_{1}
\end{aligned}
$$

Where $c_{1}=c+2\|f(1)\|$

Now put $\mathrm{y}=\mathrm{x}$ in (2.2), we get

$$
\begin{aligned}
& \left\|f\left(x^{2}\right)+f(1)-2 f(x)-2 f(x)\right\|<c \\
& \left\|f\left(x^{2}\right)-4 f(x)\right\|<c+\|f(1)\|=c_{2} \\
& \text { where } c_{2}=c+\|f(1)\| \\
& \text { Put } y=x^{2} \text { in (2.2), we get }
\end{aligned}
$$

$$
\begin{aligned}
& \left\|f\left(x^{3}\right)+f\left(x^{-1}\right)-2 f(x)-2 f\left(x^{2}\right)\right\|<c \\
& \Rightarrow\left\|f\left(x^{3}\right)-9 f(x)\right\|<c+c_{1}+2 c_{2}=c_{3}
\end{aligned}
$$

where $c_{3}=c+c_{1}+2 c_{2}$

Put $y=x^{3}$ in (2.2), we obtain

$$
\begin{aligned}
& \left\|f\left(x^{4}\right)-16 f(x)\right\|<c+4 c_{1}+c_{2}+2 c_{3}=c_{4} \text { Take } \\
& c_{4}=c+4 c_{1}+c_{2}+2 c_{3}
\end{aligned}
$$

Let $\mathrm{c}$ be as in (2.2) and define the set $\mathrm{C}$ as follows 
$C=\left\{c_{m} ; m \in N\right\}$, where $\quad c_{1}=c+2\|f(1)\|$, $c_{2}=c+\|f(1)\|$,

$$
c_{3}=c+c_{1}+2 c_{2}
$$

$c_{m}=c+(m-2)^{2} c_{1}+c_{m-2}+2 c_{m-1} \quad$ if $\mathrm{m}>3$.

Lemma 2.3: Let $f \in K Q(G ; E)$ such that

$$
\left\|f(x y)+f\left(x y^{-1}\right)-2 f(x)-2 f(y)\right\| \leq c
$$

Then for any $x \in G$ and any $m \in N$ the following relation holds

$$
\left\|f\left(x^{m}\right)-m^{2} f(x)\right\| \leq c_{m}
$$

Proof: The proof is by induction on $\mathrm{m}$. For $\mathrm{m}=3$, the lemma is established. Suppose that lemma is true for $\mathrm{m}$. Let us verified it for $m+1$. Put $y=x^{m}$ in (2.2), we have

$$
\left\|f\left(x^{m+1}\right)+f\left(x^{-m+1}\right)-2 f(x)-2 f\left(x^{m}\right)\right\|<c
$$

$\left\|f\left(x^{m+1}\right)+f\left(x^{-m+1}\right)-(m-1)^{2} f\left(x^{-1}\right)+(m-1)^{2} f\left(x^{-1}\right)-2 f(x)-2 f\left(x^{m}\right)\right\|<c$

$$
\begin{aligned}
\Rightarrow & \left\|f\left(x^{m+1}\right)+(m-1)^{2} f\left(x^{-1}\right)-2 f(x)-2 f\left(x^{m}\right)\right\| \\
& <c+\left\|f\left(x^{-1}\right)^{m-1}+(m-1)^{2} f\left(x^{-1}\right)\right\|
\end{aligned}
$$

By assumption of induction

$$
\begin{aligned}
& \left\|f\left(x^{m+1}\right)+(m-1)^{2} f\left(x^{-1}\right)-2 f(x)-2 f\left(x^{m}\right)\right\|<c+c_{m-1} \\
& \Rightarrow\left\|f\left(x^{m+1}\right)+(m-1)^{2} f\left(x^{-1}\right)-(m-1)^{2} f(x)+(m-1)^{2} f(x)-2 f(x)-2 f\left(x^{m}\right)\right\| \\
& \quad<c+c_{m-1} \\
& \Rightarrow\left\|f\left(x^{m+1}\right)+(m-1)^{2} f(x)-2 f(x)-2 f\left(x^{m}\right)\right\| \\
& \quad<c+c_{m-1}+(m-1)^{2} c_{1} \\
& \Rightarrow \| f\left(x^{m+1}\right)+(m-1)^{2} f(x)-2 f(x)-2\left[f\left(x^{m}\right)-m^{2} f(x)+m^{2} f(x) \|\right. \\
& \quad<c+c_{m-1}+(m-1)^{2} c_{1} \\
& \Rightarrow \quad\left\|f\left(x^{m+1}\right)+(m-1)^{2} f(x)-2 f(x)-2 m^{2} f(x)\right\| \\
& \quad \quad<c+c_{m-1}+(m-1)^{2} c_{1}+2 c_{m} \\
& \Rightarrow \quad\left\|f\left(x^{m+1}\right)+f(x)\left[m^{2}+1-2 m-2-2 m^{2}\right]\right\| \\
& \quad<c+c_{m-1}+(m-1)^{2} c_{1}+2 c_{m} \\
& \left\|f\left(x^{m+1}\right)-(m+1)^{2} f(x)\right\|<c+(m-1)^{2} c_{1}+c_{m-1}+2 c_{m}
\end{aligned}
$$

Now the lemma is proved.

Lemma 2.4: Let $f \in K Q(G ; E)$. For any $m>1, k \in N$ and $x \in G$, we have

$$
\left\|f\left(x^{m^{k}}\right)-\left(m^{k}\right)^{2} f(x)\right\| \leq c_{m}\left(1+m^{2}+\left(m^{2}\right)^{2}+\ldots \ldots .+\left(m^{2}\right)^{k-1}\right)
$$

and $\left\|\frac{1}{\left(m^{k}\right)^{2}} f\left(x^{m^{k}}\right)-f(x)\right\| \leq c_{m}$

Proof: The proof is based on induction on $\mathrm{k}$. If $\mathrm{k}=1$, then (2.7) follows from (2.6). Suppose that (2.7) is true for k, we will show it is true for $\mathrm{k}+1$. Replace $\mathrm{x}$ by $x^{m}$ in (2.7). $\Rightarrow$

$$
\begin{aligned}
&\left\|f\left(x^{m}\right)^{m k}-\left(m^{k}\right)^{2} f\left(x^{m}\right)\right\| \\
&<c_{m}\left(1+m^{2}+\ldots \ldots+\left(m^{2}\right)^{k-1}\right) \\
& \Rightarrow\left\|f\left(x^{m^{k+1}}\right)-\left(m^{k}\right)^{2} f\left(x^{m}\right)\right\| \\
& \quad<c_{m}\left(1+m^{2}+\ldots \ldots+\left(m^{k-1}\right)^{2}\right) \\
& \Rightarrow\left\|f\left(x^{m^{k+1}}\right)-\left(m^{k}\right)^{2} f\left(x^{m}\right)-\left(m^{k+1}\right)^{2} f(x)+\left(m^{k+1}\right)^{2} f(x)\right\| \\
& \quad<c_{m}\left(1+m^{2}+\ldots \ldots+\left(m^{k-1}\right)^{2}\right) \\
& \quad\left\|f\left(x^{m^{k+1}}\right)-\left(m^{k+1}\right)^{2} f(x)\right\| \\
& \Rightarrow<c_{m}\left(1+m^{2}+\ldots \ldots .+\left(m^{k-1}\right)^{2}\right) \\
& \quad+\left\|\left(m^{k}\right)^{2} f\left(x^{m}\right)-\left(m^{k+1}\right)^{2} f(x)\right\| \\
& \quad\left\|f\left(x^{m^{k+1}}\right)-\left(m^{k+1}\right)^{2} f(x)\right\| \\
& \Rightarrow<c_{m}\left(1+m^{2}+\ldots \ldots .+\left(m^{k-1}\right)^{2}\right)+\left(m^{k}\right)^{2} c_{m}
\end{aligned}
$$

$$
\begin{aligned}
\Rightarrow & \left\|f\left(x^{m^{k+1}}\right)-\left(m^{k+1}\right)^{2} f(x)\right\| \\
& <c_{m}\left(1+m^{2}+\ldots \ldots+\left(m^{2}\right)^{k-1}+\left(m^{2}\right)^{k}\right)
\end{aligned}
$$

Further implies

$$
\begin{aligned}
& \left(m^{k+1}\right)^{2}\left\|\frac{1}{\left(m^{k+1}\right)^{2}} f\left(x^{m^{k+1}}\right)-f(x)\right\| \\
& <c_{m}\left(1+m^{2}+\ldots \ldots+\left(m^{2}\right)^{k-1}+\left(m^{2}\right)^{k}\right) \\
& \left\|\frac{1}{\left(m^{k+1}\right)^{2}} f\left(x^{m^{k+1}}\right)-f(x)\right\| \\
& \Rightarrow \leq \frac{c_{m}}{\left(m^{2}\right)^{k+1}}\left(1+m^{2}+\ldots \ldots+\left(m^{2}\right)^{k-1}+\left(m^{2}\right)^{k}\right) \\
& \quad \leq c_{m}
\end{aligned}
$$

This completes the proof of the lemma.

From $\quad$ it follows that the set
$\left\{\frac{1}{\left(m^{k}\right)^{2}} f\left(x^{m^{k}}\right) ; k \in N\right\}$ is bounded

Replacing $\mathrm{x}$ by $x^{m^{n}}$ in (2.8), we get 


$$
\begin{aligned}
& \left\|\frac{1}{\left(m^{k}\right)^{2}} f\left(x^{m^{n+k}}\right)-f\left(x^{m^{n}}\right)\right\| \leq c_{m} \\
& \Rightarrow\left\|\frac{1}{\left(m^{n+k}\right)^{2}} f\left(x^{m^{k+1}}\right)-\frac{1}{\left(m^{n}\right)^{2}} f(x)\right\| \\
& \quad \leq \frac{c_{m}}{\left(m^{n}\right)^{2}} \rightarrow 0 \text { as } n \rightarrow \infty
\end{aligned}
$$

This implies $\quad$ that $\quad$ the sequence
$\left\{\frac{1}{\left(m^{k}\right)^{2}} f\left(x^{m^{k}}\right) ; k \in N\right\}$ is a Cauchy sequence. Since the space E is Banach. So, the above sequence has a limit in $\mathrm{E}$ and we denote it by $\phi_{m}(x)$. Thus

$$
\phi_{m}(x)=\lim _{k \rightarrow \infty} \frac{1}{\left(m^{k}\right)^{2}} f\left(x^{m^{k}}\right)
$$

From (2.8), we have

$$
\left\|\phi_{m}(x)-f(x)\right\| \leq c_{m} \quad \forall \quad x \in G
$$

Lemma 2.5: Let $f \in K Q(G ; E)$ such that

$$
\left\|f(x y)+f\left(x y^{-1}\right)-2 f(x)-2 f(y)\right\| \leq c \quad \forall x, y \in G
$$

Then for any $m \in N$, we have $\phi_{m} \in K Q(G ; E)$.

Proof: $\left\|\phi_{m}(x y)+\phi_{m}\left(x y^{-1}\right)-2 \phi_{m}(x)-2 \phi_{m}(y)\right\|$

$$
\begin{aligned}
& \left\|\phi_{m}(x y)+f(x y)+\phi_{m}\left(x y^{-1}\right)-f\left(x y^{-1}\right)-2 \phi_{m}(x)+2 f(x)\right\| \\
& -2 \phi_{m}(y)+2 f(y)+f(x y)+f\left(x y^{-1}\right)-2 f(x)-2 f(y) \| \\
& \leq\left\|\phi_{m}(x y)-f(x y)\right\|+\left\|\phi_{m}\left(x y^{-1}\right)-f\left(x y^{-1}\right)\right\|+2\left\|\phi_{m}(x)-f(x)\right\| \\
& +2\left\|\phi_{m}(y)-f(y)\right\|+\left\|f(x y)+f\left(x y^{-1}\right)-2 f(x)-2 f(y)\right\| \\
& \leq 6 c_{m}+c \\
& \left\|\phi_{m}(x y)+\phi_{m}\left(x y^{-1}\right)-2 \phi_{m}(x)-2 \phi_{m}(y)\right\|<c^{\prime},
\end{aligned}
$$

where $c^{\prime}=6 c_{m}+c$

Hence the proof of the lemma for any $x \in G$ we have the relation

$$
\phi_{m}\left(x^{m^{k}}\right)=\left(m^{k}\right)^{2} \phi_{m}(x)
$$

In fact

$$
\begin{aligned}
& \phi_{m}\left(x^{m^{k}}\right)=\lim _{l \rightarrow \infty} \frac{1}{\left(m^{l}\right)^{2}} f\left(\left(x^{m^{k}}\right)^{m^{l}}\right) \\
& =\lim _{l \rightarrow \infty} \frac{\left(m^{l}\right)^{2}}{\left(m^{l+k}\right)^{2}} f\left(x^{m^{k+l}}\right)
\end{aligned}
$$

$=\left(m^{k}\right)^{2} \lim _{p \rightarrow \infty} \frac{1}{\left(m^{p}\right)^{2}} f\left(x^{m^{p}}\right)=\left(m^{k}\right)^{2} \phi_{m}(x)$

.Lemma 2.6: If $f \in K Q(G ; E)$, then $\phi_{2} \equiv \phi_{m}$ for any $m \geq 2$.

Proof: By above lemma, $\phi_{2}, \phi_{m} \in K Q(G ; E)$.

Hence $g(x)=\lim _{k \rightarrow \infty} \frac{1}{\left(m^{k}\right)^{2}} \phi_{2}\left(x^{m^{k}}\right)$ is well defined and $g \in K Q(G ; E)$

Also $\quad g\left(x^{m^{k}}\right)=\left(m^{k}\right)^{2} g(x)$ and $g\left(x^{2^{k}}\right)=\left(2^{k}\right)^{2} g(x)$ for any $x \in G$ and any $k \in N$ also from (2.9), there exists $d_{1}, d_{2} \in R_{+}$such $\left\|\phi_{2}(x)-g(x)\right\| \leq d_{1}$ and $\left\|\phi_{m}(x)-g(x)\right\| \leq d_{2}$

Hence $g \equiv \phi_{2}$ and $g \equiv \phi_{m}$, we obtain $\phi_{2} \equiv \phi_{m}$.

Definition 2.7: By $(G ; E)$ - pseudo - Quadratic function we will mean a $(G ; E)$-quasi-Quadratic function $\mathrm{f}$ such that $f\left(x^{n}\right)=n^{2} f(x) \quad$ for any $\quad x \in G \quad$ and any $n \in Z(G ; E)$ - pseudo-Quadratic function is denoted by $P Q(G ; E)$.

Lemma 2.8: For any $f \in K Q(G ; E)$ the function $g(x)=\lim _{k \rightarrow \infty} \frac{1}{\left(2^{k}\right)^{2}} f\left(x^{2^{k}}\right)$ is well - defined and is $(G ; E)$ - pseudo - Quadratic function such that for any $x \in G,\|g(x)-f(x)\| \leq c_{2}$.

Proof: By the previous lemma, g is $a(G ; E)$ - Quasi Quadratic function. Now by above lemma, we have $g\left(x^{m}\right)=\phi_{m}\left(x^{m}\right)=m^{2} \phi_{m}(x)=m^{2} g(x)$

Thus $\phi_{m}(x)=g(x)$ and hence $\phi_{2}(x)=g(x)$ by above lemma. From equality

$g=\phi_{2}$, we have $\|g(x)-f(x)\|$

$=\left\|\phi_{2}(x)-f(x)\right\| \leq c_{2}$.

Lemma 2.9: If $f \in P Q(G ; E)$ then

(i) If $y \in G$ is an element of finite order then $f(y)=0$.

(ii) If $\mathrm{f}$ is bounded function on $\mathrm{G}$, then $f \equiv 0$.

Proof: Let order of $y \in G$ is t then 


$$
\begin{array}{ll} 
& y^{t}=1 \\
\Rightarrow \quad & f\left(y^{t}\right)=f(1)=0 \\
& t^{2} f(y)=0 \\
\Rightarrow & f(y)=0 .
\end{array}
$$

Similarly we verify assertion 2 .

We denoted by $B(G ; E)$ the space of all bounded functions on a group $\mathrm{G}$ that takes values in $\mathrm{E}$.

Theorem 2.10: For an arbitrary group $G$ the following decomposition holds:

$$
K Q(G ; E)=P Q(G ; E) \oplus B(G ; E) .
$$

Proof: It is clear $P Q(G ; E)$ and $B(G ; E)$ are subspaces of $K Q(G ; E), \quad$ and $P Q(G ; E) \cap B(G ; E)=\{0\}$. Hence the subspace of $K Q(G ; E) \quad$ generated by $P Q(G ; E)$ and $B(G ; E)$ is their direct sum.

That is $P Q(G ; E) \oplus B(G ; E) \subseteq K Q(G ; E)$.

Now let $f \in K Q(G ; E)$, then $g \in P Q(G ; E)$ and $g-f \in B(G ; E)$

and

$f=g+(g-f) \in P Q(G ; E)+B(G ; E)$

So, $K Q(G ; E)=P Q(G ; E) \oplus B(G ; E)$.

Remark2.11: Let $\mathrm{G}=\{1,-1, \mathrm{i},-\mathrm{i}\}$ be an abelian group and the quadratic functional equation is not stable in $G$ i.e, $f(x y)+f\left(x y^{-1}\right)=2 f(x)+2 f(y)$ does not satisfy for $\mathrm{x}=-1, \mathrm{y}=-1$.

Definition2.12: We shall say that equation (2.1) is stable for the pair $(\mathrm{G} ; \mathrm{E})$ if for any $\mathrm{f}: \mathrm{G} \rightarrow \mathrm{E}$ satisfying functional inequality

$$
\begin{gathered}
\left\|f(x y)+f\left(x y^{-1}\right)-2 f(x)-2 f(y)\right\| \leq c \\
\forall \mathrm{x}, \mathrm{y} \in \mathrm{G}
\end{gathered}
$$

for some $\mathrm{c}>0$, there is a solution $\bar{f}$ of the functional equation (2.1) such that the function $\bar{f}(\mathrm{x})-\mathrm{f}(\mathrm{x})$ belongs to $\mathrm{B}(\mathrm{G} ; \mathrm{E})$.

It is clear that equation (2.1) is stable on $\mathrm{G}$ iff PQ $(\mathrm{G} ; \mathrm{E})=\mathrm{Q}_{0}$ $(\mathrm{G}$; E). We will say that a $(\mathrm{G}$; E) - pseudo - quadratic function $\mathrm{f}$ is nontrivial if $\mathrm{f} \notin \mathrm{Q}_{0}(\mathrm{G}$; $\mathrm{E})$

Theorem2.13: Let $E_{1}, E_{2}$ be a branch space over real. Then equation (2.1) is stable for the pair $\left(G ; E_{1}\right)$ if and only if it is stable for the pair $\left(\mathrm{G} ; \mathrm{E}_{2}\right)$.

Proof: $\rightarrow$ Let $\mathrm{E}$ be a branch space and R be the set of real . Suppose that equation (2.1) is stable for the pair (G, E). Suppose that (2.1) is not stable for the pair $(G ; R)$, then there is a non trivial real -valued pseudo - quadratic function $\mathrm{f}$ on G. Now let $\mathrm{e} \in \mathrm{E}$ and $\|e\|=1$. Consider the function $\phi: \mathrm{G} \rightarrow$ E given by the formula $\phi(x)=\mathrm{f}(\mathrm{x})$.e. It clears that $\phi$ is a nontrivial pseudo -quadratic E-valued function, and we obtain a contradiction.

Now suppose that equation (2.1) is stable for the pair $(G ; R)$, that is , $P Q(G ; R)=Q_{0}(G ; R)$. Denote by $E^{*}$ the space of linear bounded functional on $\mathrm{E}$ endowed by functional norm topology. It is clear that for any $\psi \in \mathrm{PQ}(\mathrm{G} ; \mathrm{H})$ and $\lambda \in$ $\mathrm{H}^{*}$ the function $\lambda 0 \psi$ belongs to the space $\mathrm{PQ}(\mathrm{G} ; \mathrm{R})$. Indeed, let for some $c>0$ and any $x, y \in G$ we have

$$
\left\|\psi(x y)+\psi\left(x y^{-1}\right)-2 \psi(x)-2 \psi(y)\right\| \leq c .
$$

Hence

$$
\begin{aligned}
& \left|\lambda o \psi(x y)+\lambda o \psi\left(x y^{-1}\right)-\lambda o \psi(2 x)-\lambda o \psi(2 y)\right|= \\
& \mid \lambda\left[\psi(x y)+\psi\left(x y^{-1}\right)-2 \psi(x)-2 \psi(y)\right] \leq c\|\lambda\|
\end{aligned}
$$

Obviously, $\lambda o \psi\left(x^{n}\right)=n^{2} \lambda o \psi(x)$ any $\mathrm{x} \in \mathrm{G}$ ad for any $\mathrm{n} \in \mathrm{N}$. Hence the function $\lambda o \psi$ belong to the space $\mathrm{PQ}(\mathrm{G}, \mathrm{R})$. Let $\mathrm{f}: \mathrm{G} \rightarrow \mathrm{H}$ be a nontrivial pseudo- Quadratic mapping. Then $\mathrm{x}, \mathrm{y} \in \mathrm{G}$ such that $f(x y)+f\left(x y^{-1}\right)-2 f(x)-2 f(y) \neq 0 . \quad$ HahnBanach theorem implies that there is a $l \in H^{*}$ such that $l\left(f(x y)+f\left(x y^{-1}\right)-2 f(x)-2 f(y)\right) \neq 0$, and we have lof is a nontrivial pseudo- Quadratic real valued function on $\mathrm{G}$. This contradiction proves the theorem.

Corollary2.14: If a group $\mathrm{G}$ has nontrivial pseudo- character, then equation (2.1) is not stable on G.

Proof: Let $\phi$ be a nontrivial pseudo- character of G. Suppose that there is $\bar{f} \in \mathrm{Q}_{0}(\mathrm{G})$ such that the function $\phi-\bar{f}$ is bounded. Then there is $c>0$ such that $|\phi(x)-\bar{f}(x)| \leq c$ for any $\mathrm{x} \in \mathrm{G}$. Hence for any $\mathrm{n} \in \mathrm{N}$ we have $c \geq\left|\phi\left(x^{n}\right)-\bar{f}\left(x^{n}\right)\right|=n^{2}|\phi(x)-\bar{f}(x)|$ and we see that the latter is possible if $\phi(x)=\bar{f}(x)$. So, $\phi \in P Q(G) \cap Q_{0}(G)$. Hence, $f \in X(G)$ and this contradiction with the assumption about $\mathrm{f}$.

\section{REFERENCES}

[1] Aoki,T., (1950) "On stability of the linear transformation in Banach spaces," Journal of the Mathematical Society of Japan, 2,64-66

[2] Chang, S. and Kim,H.M., (2002), On the Hyer-Ulam stability of a quadratic functional equations, J.Ineq. Appl. Math., 33, 1-12.

[3] Chang,S., Lee,E.H. and Kim,H.M., (2003)On the HyerUlam Rassias stability of a quadratic functional equations, Math. Ineq. Appl. Math., 6(1), 87-95.

[4] Cholewa,P.W (1984) Remarks on the stability of functional equations, Aequationes Math., 27, 76-86. 
[5] Czerwik,S., (1992),On the stability of a quadratic mappings in normed spaces, Abh. Math.Sem. Univ. Hamburg.,62, 59-64.

[6] Gavruta,P., (1994) A generalization of the Hyers-UlamRassias stability of approximately additive mappings, J.Math. Anal. Appl., 184,431-436.

[7] Gruber,P.M., (1978), Stability of isometries, Trans. Amer. Math. Soc. 245, 263-277.

[8] Hyers,D.H. and Rassias,T.M., (1992) Approximate homomorphism, Aequationes Math., 44,125-153.

[9] Hyers,D.H., Isac, G., and Rassias, Th.M., (1998), Stability of functional equations in several variables, Birkhauser Basel.

[10] Hyers,D.H., (1941) On the stability of the linear functional equation, Proc. Nat. Acad. Sci. U.S.A., 27, 222-223.
[11] Hyers,D.H., Isac, G. and Rassias,Th.M., (1998) On the asymptotically of Hyer-Ulam stability of mappings, Proc. Amer. Math. Soc., 126),425-430.

[12] Jang,S.Y., Lee,J.R., Park,C. and Shin,D.Y., (2009)Fuzzy stability of Jensen-type Quadratic functional equations, Abstract and Applied Analysis, pp-17.

[13] Mirzavaziri,M. and Moslehian,M.S., A fixed point approach to stability of Quadratic equation, arXivimath/0512007v1 [math.FA] 1 Dec 2005.

[14] Rassias,T.M., (1978) On stability of linear mapping in Banach spaces, Proc. Amer. Math. Soc. 72, 297-300.

[15] SKOF,F., (1983) Proprieta localie approssimazione di operatori, Rend. Sem. Mat. Fis. Milano, 53, 113-129

[16] Ulam,S.M., (1960) A collection of Mathematical Problems, Interscience tracts in Pure and Applied Mathematics, U.S.A 16.

[17] Ulam,S.M., 1963, Problems in Modern Mathematics, John Wiley \& Sons, New York, USA,. 